\title{
BMPs on the road to hepatogenesis
}

\begin{abstract}
Stephen A. Duncan ${ }^{1}$ and Alistair J. Watt
Classical transplantation studies using chick and quail embryos demonstrated that the vertebrate liver parenchyma is derived from an outgrowth of the ventral foregut endoderm, referred to as the liver bud (LeDouarin 1964, 1968, 1975). This process, illustrated in Figure 1, initiates at around gestation day 8.25 (7-8 somites) in the mouse (Gualdi et al. 1996). Invagination of the foregut positions a ventral portion of foregut endoderm next to the developing cardiac mesoderm. Early on, it was proposed that specification of the hepatic diverticulum relied upon the close proximity of cardiac mesoderm. Several studies, the most recent by Gualdi et al. (1996), provided strong evidence to support this proposal. In general, it was found that ventral endoderm from either mouse or chick was unable to adopt a hepatic fate, or express characteristic hepatic mRNAs, if it were grown in the absence of developing cardiac tissue (LeDouarin 1975; Houssaint 1980; Fukuda-Taira 1981; Gualdi et al. 1996). Moreover, this inductive capacity seemed to be restricted to cardiac mesoderm, because other noncardiac mesoderms failed to induce hepatic development (Fukuda 1979; Fukuda-Taira 1981).
\end{abstract}

Department of Cell Biology, Neurobiology and Anatomy, Medical College of Wisconsin, Milwaukee, Wisconsin 53226, USA

\section{Not so lonely heart}

Although it was firmly established that the developing heart played an important inductive role during hepatogenesis, the molecular mechanisms underlying this activity were defined only recently, largely through work carried out by the Zaret laboratory (Gualdi et al. 1996; Jung et al. 1999). Jung et al. (1999) used a tissue explant assay to demonstrate that fibroblast growth factors (FGFs) 1 or 2 could substitute for cardiac mesoderm in inducing ventral endoderm to elicit a hepatogenic response. In these experiments expression of albumin mRNA, a marker of nascent hepatic cells, could be detected within ventral endoderm that had been isolated from embryos prior to specification of the liver (2-6 somites) and cultured for $2 \mathrm{~d}$ either in the presence of cardiac tissue or in the presence of FGF1 or FGF2. In contrast, when prehepatic ventral endoderm was cultured alone it failed to express albumin mRNA. These data strongly supported a model wherein FGFs secreted from the developing heart were sufficient to induce the

${ }^{1}$ Corresponding author.

E-MAIL duncans@mcw.edu; FAX (414) 456-6517.

Article and publication are at http://www.genesdev.org/cgi/doi/10.1101/ gad.920601. onset of hepatic development. However, the time-honored belief that hepatic induction is solely a matter of the heart has now been overturned by further work from the Zaret lab (Rossi et al. 2001, this issue), showing that bone morphogenic proteins (BMPs) secreted from septum transversum mesenchyme are needed in concert with cardiac-derived FGFs to induce the ventral endoderm to adopt a hepatic fate.

The septum transversum mesenchyme remains closely associated with the liver throughout development and ultimately contributes toward the diaphragm and epicardium. Following specification of the hepatic lineage, hepatoblasts rapidly proliferate and migrate away from the foregut into the adjacent septum transversum mesenchyme (Fig. 1). Using chick/quail grafts and coculture experiments with mouse tissues, it was found that hepatoblasts require an undefined signal from the septum transversum mesenchyme to complete their differentiation into mature hepatocytes (LeDouarin 1968; Houssaint 1980). However, this signal to differentiate was not restricted to the septum transversum, because hepatic differentiation could also be induced by culturing hepatic endoderm with heterologous mesenchymes (Houssaint 1980). The possibility that septum transversum mesenchyme could also contribute toward the distinct act of specifying the hepatic lineage had not previously been considered, because it was believed that only cardiac tissue was required. However, Rossi et al. (2001) have now addressed this possibility by taking advantage of a transgenic mouse in which expression of a $\mathrm{LacZ}$ reporter gene is controlled by the endogenous $B m p 4$ transcriptional regulatory elements (Lawson et al. 1999|. The LacZ expression pattern in this mouse mimicked that of endogenous BMP4, including expression in the septum transversum but not in the ventral foregut endoderm. Importantly, the authors identified LacZ expressing septum transversum mesenchyme that closely apposed the ventral endoderm around the time of hepatic specification (8 somites). This implied, through guilt by association, that the septum transversum might act in a juxtacrine fashion to influence hepatic specification. Moreover, the fact that BMP4 was expressed in the septum transversum raised the possibility that BMPs could mediate the proposed inductive action.

\section{BMPs and FGFs act in concert}

An essential role for BMP signaling was demonstrated directly by culturing the prehepatic ventral endoderm 
Figure 1. FGFs and BMPs in liver development. Sagittal sections through embryos at $8.25 \mathrm{~d}$ postcoitum (dpc) (upper left) and $9.5 \mathrm{dpc}$ (lower left), with each area undergoing hepatogenesis boxed and shown in detail on the right. By this stage (2-6 somite stage), invagination of the foregut endoderm positions a portion of ventral endoderm (green) adjacent to the cardiac mesoderm (red) and the septum transversum mesenchyme (blue). At this stage, the entire endoderm expresses the transcription factor Hnf3, making the endoderm competent to adopt a hepatic fate. BMP signaling from the septum transversum and FGF signaling from the cardiac mesoderm induce a portion of the ventral endoderm to initiate hepatogenesis (green), leaving the distal lip of endoderm to form the ventral pancreas (yellow). This results in the expression of transcription factors such as Hex and Gata4 necessary for subsequent developmental stages. By $8.5 \mathrm{dpc}$ the hepatic endoderm thickens to form the liver bud. The continued action of BMPs and FGFs is required for the proliferation and outgrowth of the liver bud. By 9.5 dpc Hnf4 $\alpha$, which is necessary to complete hepatocyte differentiation, is expressed in the migrating hepatic cells.

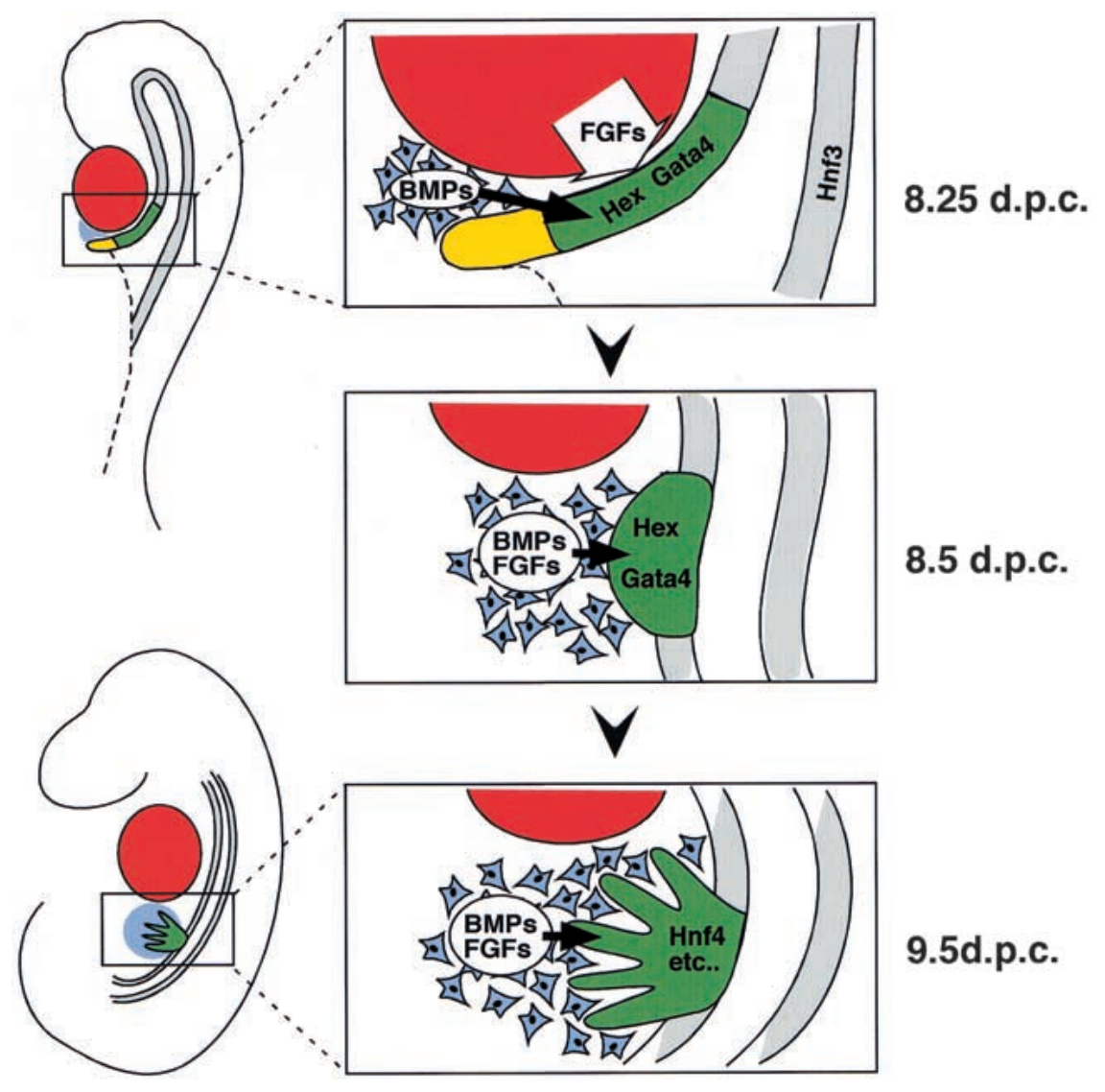

with cardiac and septum transversum tissue in the presence of the Xenopus laevis noggin protein (Xnoggin). Xnoggin acts as a potent antagonist of BMP action, and its presence in the media of cultured explants was found to inhibit the onset of albumin mRNA expression within the ventral endoderm relative to control cultures lacking Xnoggin. The demonstration that BMPs were necessary for hepatic specification raised the question of why, in previous experiments, FGF alone was capable of inducing albumin mRNA expression in cultured ventral endoderm. The authors suggest that it is likely, in retrospect, that the ventral endoderm isolates contained BMP-expressing septum transversum mesenchyme. Indeed, examination of cultured endoderm explants revealed mesenchymal cells that expressed the septum transversum marker Mrg1 as well as BMP4. The authors also found that Xnoggin inhibited FGF-induced expression of albumin mRNA in cultured ventral endoderm. This result was illuminating because it also demonstrated that FGF alone was insufficient to induce the onset of a hepatic gene expression program within the ventral endoderm. Similarly, explants cultured in the presence of exogenous BMPs but in the absence of cardiac tissue or FGF also failed to express albumin mRNA. These results prompt a model in which at least two signaling pathways originating from different tissue sources, FGF (cardiac mesoderm) and BMP (septum transversum mesenchyme), must converge at the ventral endoderm to drive the onset of hepatogenesis.
This model is appealing on a number of levels. The fact that two tissues must contribute signals that induce hepatogenesis reinforces the dynamic, multidimensional nature of embryogenesis. It means, simply, that a tissue that is competent to follow a given fate must be positioned in the right place at the right time to fulfill that fate. In this regard, the identification of FGF and BMPs as inducers of hepatic development define a mechanism whereby outgrowth of the liver is positioned along the anterior-posterior of the ventral foregut. In addition to deciding the position of liver development, it is likely that the combined action of FGF and BMP signaling defines the domain of endoderm that gives rise to the ventral pancreas. Like the liver, ventral pancreas also originates as a budding outgrowth of the ventral endoderm. Deutsch et al. (2001) recently demonstrated, again using explant cultures, that the liver and ventral pancreatic domains are nonoverlapping, that is, the ventral pancreas derives from a lip of endoderm that extends beyond the immediate vicinity of the developing heart (Fig. 1). Further experiments showed that the entire ventral endoderm, including the portion fated to become liver, is innately capable of adopting a pancreatic fate by default. Indeed, when ventral endoderm that would normally follow a hepatic fate is cultured in the absence of cardiac mesoderm or FGF, it initiates a gene expression program that is characteristic of the developing pancreas. However, if FGF or cardiac mesoderm is included in the culture medium, the fate of the endoderm is diverted from 
pancreatic to hepatic. Originally it was proposed that the anteriormost portion of endoderm fails to adopt a hepatic fate because it encounters levels of FGFs that are insufficient to induce hepatogenesis (Deutsch et al. 2001). The current work by Rossi et al. (2001) extends this model: Culture of endoderm in the presence of Xnoggin also resulted in expression of the pancreatic marker $P d x 1$, suggesting that BMP acts in concert with FGF to exclude the pancreatic fate of the ventral endoderm.

\section{BMPs and the liver bud}

Bud formation is a central theme in organogenesis that usually involves interactions between mesenchymal and epithelial cells. Using the definition of Hogan (1999), a primary bud is a "knob-like cluster of progenitor cells located within distinct boundaries that proliferate and move away from the surface of a pre-existing structure." Limbs, genitalia, pancreas, thyroid, lung, liver, branchial arches, and even feathers in avian species are included in the list of organs that initiate their development through the formation of a primary bud. In almost all cases, now including the liver, the combined action of BMPs and FGFs is important for bud initiation (for review, see Hogan 1999). Formation of a primary bud can be divided into discrete stages. First, the site of bud formation is defined within the axis of the developing embryo, and regions of secreted signaling molecules are established. In the case of the liver bud, morphogenic movements ultimately position the ventral endoderm next to the cardiac mesoderm and septum transversum mesenchyme. This places the presumptive hepatic endoderm within an environment of defined signaling factors, namely FGFs and BMPs, and establishes its location within the anterior-posterior, dorsal-ventral, and medial-lateral axes of the embryo.

The second phase of primary bud formation requires the proliferation of cells in response to mitogens and growth factors with an accompanied morphogenic outgrowth of the bud. This is also true for formation of the liver diverticulum-shortly after induction, the nascent hepatic cells proliferate, then delaminate and migrate away from the constraints of the foregut (see Fig. 1). Expansion and migration of hepatoblasts also occurs in explant cultures (Gualdi et al. 1996; Jung et al. 1999). Moreover, as the hepatic cells migrate they are accompanied by septum transversum mesenchyme as is observed in vivo (Rossi et al. 2001). Recently it has been shown that the migration of the hepatoblasts during this stage of development is dependent upon the transcription factor Prox1 (Sosa-Pineda et al. 2000). In addition, Jung (1999) showed that FGF8 also contributes toward the morphogenic outgrowth of the liver, although they postulated that it works in conjunction with a second undefined signal. The current work from the Zaret lab has demonstrated a direct role for BMP signaling in the outgrowth of the hepatic bud. They showed that when explants consisting of cardiac mesoderm, septum transversum, and ventral endoderm isolated from wild-type, $\mathrm{Bmp}^{+/-}$, or $B m p 4^{-/-}$embryos were cultured for $2 \mathrm{~d}$, endodermal cells were seen to flatten morphologically and migrate away from the beating cardiac cells. However, if Xnoggin was added to the media, $B m p 4^{+/-}$or $B m p 4^{-/-}$endodermal cells failed to migrate, and the cells remained compact and rounded. This implies that, in addition to specification, BMPs are required for the morphogenic expansion of hepatic cells during liver bud formation. Surprisingly, in contrast to $B \mathrm{mp} 4^{+/-}$or $B m p 4^{-/-}$explants, cell outgrowth from wild-type explants was unaffected by the inclusion of Xnoggin. This may be due to an inability of Xnoggin to completely penetrate the cultured tissues and/or antagonize the levels of BMP found in wild-type compared to $\mathrm{Bmp}^{+/-}$, or $\mathrm{Bmp4}^{-/-}$explants. Whether FGF and/or BMPs directly affect cell proliferation or are specifically required for delamination and migration awaits further analysis. However, the known mitogenic activity of FGFs in other developmental systems such as limb and lung bud formation would be consistent with a role for FGFs in directing cell proliferation within the liver bud (Szebenyi and Fallon 1999).

The environment in which the ventral endoderm finds itself is obviously key in determining its fate. FGFs are short-range effectors because their high affinity for extracellular matrix components, for example, heparin sulfate moieties, holds them close to the site of secretion (Szebenyi and Fallon 1999). For this reason, the endoderm must juxtapose the cardiac mesoderm if hepatic specification is to occur. In contrast, BMPs appear capable of acting at greater distances from their site of secretion. In addition, ample evidence exists that BMPs can act as morphogens, in that they are capable of inducing multiple cell fates depending upon their concentration. The most direct evidence supporting the view of BMPs as morphogens comes from the Hemmati-Brivanlou group (Wilson and Hemmati-Brivanlou 1995; Wilson et al. 1997). When $X$. laevis animal caps are dissociated and cultured in the absence of BMP4, the ectoderm adopts a neural fate, and when cultured in high BMP4 concentrations, it follows an epidermal fate. However, if the explants are cultured in intermediate concentrations of BMP4, the ectoderm expresses genes that are characteristic of ectodermal-derived cement gland tissue. This raises the question of whether a unique concentration of BMPs is necessary to specify a hepatic fate within the ventral endoderm or whether simply its presence in conjunction with FGF is sufficient. In addition to putative concentration gradients, the developing embryo can modulate BMP activity through the expression of a variety of $\mathrm{BMP}$ anatgonists (for review, see Massague and Chen 2000). In addition, noggin, follistatin, chordin, and the DAN family of proteins, including Cerberus, have all been shown to antagonize BMP signaling. Injection of Cerberus mRNA into $X$. laevis embryos has been found to induce a dramatic duplication of both the heart and liver (Bouwmeester et al. 1996). This result seems to contradict the finding that BMPs induce hepatic specification. However, it is possible that the formation of ectopic livers in these experiments is a secondary consequence of the duplication of the heart. In this case, it may be predicted that the duplicated cardiac tissue sets 
up a new field of FGF expression, which, in turn, induces a second liver bud. Regardless, the existence of modulators of BMP activity clearly raises the possibility that they could contribute toward defining the hepatic domain within the ventral endoderm.

\section{What's next-transcription?}

The differentiated phenotype of any cell is ultimately defined by gene expression. It would, therefore, seem logical that transcription factors play key roles in deciding the fate of a progenitor cell and that expression of hepatic transcription factor genes is modulated through FGF and BMP signaling. Work from several laboratories has recently expanded the repertoire of transcription factors with known roles in liver development (for review, see Duncan 2000). Particularly striking is the apparent absence of a liver in midgestation mouse embryos lacking Hex (Keng et al. 2000; Martinez Barbera et al. 2000). Hex is a homeodomain transcription factor that is expressed in the ventral endoderm around the time a liver fate is specified (Keng et al. 1998; Thomas et al. 1998). Examination of $\mathrm{Hex}^{-/-}$embryos at gestation day $9.5 \mathrm{re}-$ vealed an arrest in formation of the liver bud and is the earliest hepatic developmental defect so far described. Analysis of hepatic gene expression in $\mathrm{Hex}^{-/-}$embryos revealed the onset of expression of the liver transcription factors $H n f 1 \alpha, H n f 4 \alpha$, and $H n f 6$ within the ventral endoderm even although no liver bud had formed. This implies that although $\mathrm{Hex}^{-/-}$endoderm is competent to respond to FGF and BMP induction, it cannot complete the earliest stages of liver development (Keng et al. 2000). This loss of the hepatic lineage so early in development suggests Hex is a good candidate target of BMP or FGF signaling. Recent analysis of the Hex promoter has identified elements required for expression of a $L a c Z$ transgene in the developing livers of transgenic mice (Rodriguez et al. 2001). However, further experiments are required to determine whether these elements can be activated in response to FGF or BMPs. The current work of Rossi et al. (2001) identified a second transcription factor, Gata4, as a possible target of BMP action. Gata4 has been implicated as having a role in early hepatic development through its occupancy of the albumin promoter within the developing endoderm (Bossard and Zaret 1998). When ventral endoderm explants are cocultured with cardiac mesoderm and septum transversum mesenchyme, Gata4 mRNA can be detected within the nascent hepatic endoderm by in situ hybridization. However, when Xnoggin is included in the culture medium, no Gata4 mRNA is detected. Such a result is consistent with Gata4 acting downstream of BMP action. Unfortunately, foregut development is severely defective in Gata4 $^{-1-}$ embryos, and this, so far, has prevented the use of these embryos to directly determine the role of Gata4 during hepatogenesis (Kuo et al. 1997; Molkentin et al. 1997). However, both Gata4 and the related factor Gata6 are essential for development of extraembryonic visceral endoderm. This may be significant given that the visceral endoderm and liver share many characteristics such as expression of several common transcription factors including $H n f 1 \alpha, H n f 1 \beta$, Gata factors, the Hnf3 family, and $H n f 4 \alpha$ (Duncan et al. 1998). Furthermore, loss of $\mathrm{Hnf} 4 \alpha$ function disrupts differentiation of both visceral endoderm and liver, suggesting that the two tissues use common developmental pathways (Chen et al. 1994; Duncan et al. 1997; Li et al. 2000). The finding that Gata factors can directly transactivate expression from the $H n f 4 \alpha$ promoter implies that they act upstream of Hnf4 $\alpha$ during hepatogenesis (Morrisey et al. 1998). If this is true and if, indeed, Gata4 or Gatab is a target of BMP signaling, it would describe a chain of molecular events linking induction of hepatogenesis by BMP to subsequent differentiation through the regulation of Hnf4 expression by Gata factors.

\section{Being prepared: Hnf3 as a competency factor}

Most signaling factors with clear roles in developmental processes can be characterized as members of one of the FGF, BMP/TGF- $\beta$, EGF, Hedgehog, or Wnt multigene families. Although each family has multiple members, it is clear that these molecules are used over and over again throughout embryogenesis. This provokes the question of how the same factors, and often the same combination of factors, regulate such a range of developmental responses. The obvious answer would be that specific responses are decided at the level of the receptor and the intracellular signaling mechanisms that act downstream of ligand binding. While this clearly plays a role, it is also true that a given tissue must be competent to respond to the signal. Classically, competency is defined as the inherent ability of a tissue to progress toward a defined fate if appropriately induced (Waddington 1940). In the case of the developing liver (and this is likely to be true for other organs), competency requires the expression of specific transcription factors. The Hnf3 winged-helix factors, $\mathrm{Hnf} 3 \alpha$ and $\mathrm{Hnf} 3 \beta$, are expressed throughout the definitive endoderm prior to the onset of liver development (Ang et al. 1993; Monaghan et al. 1993; Ruiz i Altaba et al. 1993; Sasaki and Hogan 1993). Gualdi et al. (1996) found that Hnf3 bound the albumin enhancer throughout the definitive endoderm, and therefore marked the transcriptionally inactive albumin gene, prior to the onset of hepatic development. Hnf3 has a higher affinity for its binding site in the context of nucleosomal DNA than in naked plasmid DNA (Cirillo and Zaret 1999). In addition, it has the capacity to relieve chromatin-mediated repression of gene expression as well as the ability to reposition nucleosomes within the albumin enhancer (Shim et al. 1998; Crowe et al. 1999). This implies a model wherein Hnf3 maintains the competency of endoderm to adopt a hepatic fate by optimizing chromatin structure around the enhancers of genes expressed in the liver anlage, making them accessible to transcriptional activators. FGF and BMP action could then activate expression of transcription factors such as Gata4 and Hex, which would then have access to Hnf3-marked enhancers and could induce a hepatic differentiation program (Fig. 1). 
As always, breakthroughs lead to more questions than answers, and the discovery of the role of BMPs in regulating liver development is no exception. However, this elegant work by the Zaret laboratory (Rossi et al. 2001) also provides a window through which we can begin to view the molecular underpinnings of hepatogenesis and organogenesis.

\section{Acknowledgments}

We thank Paula Traktman and Wendy Garrison for critically reading the manuscript and offering helpful suggestions and to Pamela Hoodless for advice on BMP inhibitors.

\section{References}

Ang, S.-L., Wierda, A., Wong, D., Stevens, K.A., Cascio, S., Rossant, J., and Zaret, K.S. 1993. The formation and maintenance of the definitive endoderm lineage in the mouse: Involvement of HNF-3/forkhead proteins. Development 119: 1301-1315.

Bossard, P. and Zaret, K.S. 1998. GATA transcription factors as potentiators of gut endoderm differentiation. Development 125: 4909-4917.

Bouwmeester, T., Kim, S., Sasai, Y., Lu, B., and De Robertis, E.M. 1996. Cerberus is a head-inducing secreted factor expressed in the anterior endoderm of Spemann's organizer. Nature 382: 595-601.

Chen, W.S., Manova, K., Weinstein, D.C., Duncan, S.A., Plump, A.S., Prezioso, V.R., Bachvarova, R.F., and Darnell, J.E., Jr. 1994. Disruption of the HNF-4 gene, expressed in visceral endoderm, leads to cell death in embryonic ectoderm and impaired gastrulation of mouse embryos. Genes \& Dev. 8: 2466-2477.

Cirillo, L.A. and Zaret, K.S. 1999. An early developmental transcription factor complex that is more stable on nucleosome core particles than on free DNA. Mol. Cell 4: 961-969.

Crowe, A.J., Sang, L., Li, K.K., Lee, K.C., Spear, B.T., and Barton, M.C. 1999. Hepatocyte nuclear factor 3 relieves chromatinmediated repression of the $\alpha$-fetoprotein gene. J. Biol. Chem. 274: 25113-25120.

Deutsch, G., Jung, J., Zheng, M., Lora, J., and Zaret, K.S. 2001. A bipotential precursor population for pancreas and liver within the embryonic endoderm. Development 128: 871-881.

Duncan, S.A. 2000. Transcriptional regulation of liver development. Dev. Dyn. 219: 131-142.

Duncan, S.A., Nagy, A., and Chan, W. 1997. Murine gastrulation requires HNF-4 regulated gene expression in the visceral endoderm: Tetraploid rescue of HNF-4-/- embryos. Development 124: 279-287.

Duncan, S.A., Navas, A.M., Dufort, D., Rossant, J., and Stoffel, M. 1998. Regulation of a transcription factor network required for cell differentiation and metabolism. Science 281: 692-695.

Fukuda, S. 1979. The development of hepatogenic potency in the endoderm of quail embryos. J. Embryol. Exp. Morphol. 52: 49-62.

Fukuda-Taira, S. 1981. Hepatic induction in the avian embryo: Specificity of reactive endoderm and inductive mesoderm. $J$. Embryol. Exp. Morphol. 63: 111-125.

Gualdi, R., Bossard, P., Zheng, M., Hamada, Y., Coleman, J.R., and Zaret, K.S. 1996. Hepatic specification of the gut endoderm in vitro: Cell signaling and transcriptional control. Genes \& Dev. 10: 1670-1682.
Hogan, B.L. 1999. Morphogenesis. Cell 96: 225-233.

Houssaint, E. 1980. Differentiation of the mouse hepatic primordiam. I. An analysis of tissue interactions in hepatocyte differentiation. Cell. Diff. 9: 269-279.

Jung, J., Zheng, M., Goldfarb, M., and Zaret, K.S. 1999. Initiation of mammalian liver development from endoderm by fibroblast growth factors. Science 284: 1998-2003.

Keng, V.W., Fujimori, K.E., Myint, Z., Tamamaki, N., Nojyo, Y., and Noguchi, T. 1998. Expression of Hex mRNA in early murine postimplantation embryo development. FEBS Lett. 426: $183-186$.

Keng, V.W., Yagi, H., Ikawa, M., Nagano, T., Myint, Z., Yamada, K., Tanaka, T., Sato, A., Muramatsu, I., Okabe, M., et al. 2000. Homeobox gene Hex is essential for onset of mouse embryonic liver development and differentiation of the monocyte lineage. Biochem. Biophys. Res. Commun. 276: $1155-1161$

Kuo, C.T., Morrisey, E.E., Anandappa, R., Sigrist, K., Lu, M.M., Parmacek, M.S., Soudais, C., and Leiden, J.M. 1997. GATA4 transcription factor is required for ventral morphogenesis and heart tube formation. Genes \& Dev. 11: 1048-1060.

Lawson, K.A., Dunn, N.R., Roelen, B.A., Zeinstra, L.M., Davis, A.M., Wright, C.V., Korving, J.P., and Hogan, B.L. 1999. BMP4 is required for the generation of primordial germ cells in the mouse embryo. Genes \& Dev. 13: 424-436.

LeDouarin, N. 1964. Etude experimentale de l'organogenese du tube digestif et du foie chez l'embryon de poulet. Bull. Biol. Fr. Belg. 98: 543-676.

1968. Synthese du glycogene dans les hepatocytes en voie de differentiation: Role des mesenchymes homologue et heterologues. Dev. Biol. 17: 101-114.

. 1975. An experimental analysis of liver development. Med. Biol. 53: 427-455.

Li, J., Ning, G., and Duncan, S.A. 2000. Mammalian hepatocyte differentiation requires the transcription factor HNF-4 $\alpha$. Genes \& Dev. 14: 464-474.

Martinez Barbera, J.P., Clements, M., Thomas, P., Rodriguez, T., Meloy, D., Kioussis, D., and Beddington, R.S. 2000. The homeobox gene Hex is required in definitive endodermal tissues for normal forebrain, liver and thyroid formation. Development 127: 2433-2445.

Massague, J. and Chen, Y.G. 2000. Controlling TGF- $\beta$ signaling. Genes \& Dev. 14: 627-644.

Molkentin, J.D., Lin, Q., Duncan, S.A., and Olson, E.N. 1997. Requirement of the transcription factor GATA4 for heart tube formation and ventral morphogenesis. Genes \& Dev. 11: 1061-1072.

Monaghan, A.P., Kaestner, K.H., Grau, E., and Schutz, G. 1993. Postimplantation expression patterns indicate a role for the mouse forkhead/HNF- $3 \alpha, \beta$, and $\gamma$ genes in determination of the definitive endoderm, chordamesoderm, and neurectoderm. Development 119: 567-578.

Morrisey, E.E., Tang, Z., Sigrist, K., Lu, M.M., Jiang, F., Ip, H.S., and Parmacek, M.S. 1998. GATA6 regulates HNF4 and is required for differentiation of visceral endoderm in the mouse embryo. Genes \& Dev. 12: 3579-3590.

Rodriguez, T.A., Casey, E.S., Harland, R.M., Smith, J.C., and Beddington, R.S. 2001. Distinct enhancer elements control hex expression during gastrulation and early organogenesis. Dev. Biol. 234: 304-316.

Rossi, J.M., Dunn, R.N., Hogan, B.L.M., and Zaret, K.S. 2001. Distinct mesodermal signals, including BMPs from septum transversum mesenchyme, are required in combination for hepatogenesis from the endoderm. Genes \& Dev. 15: 19982009 (this issue).

Ruiz i Altaba, A., Prezioso, V.R., Darnell, J.E., and Jessell, T.M. 
1993. Sequential expression of HNF3 $\beta$ and HNF3 $\alpha$ by embryonic organizing centers: The dorsal lip/node, notochord and floor plate. Mech. Dev. 44:91-108.

Sasaki, H. and Hogan, B.L.M. 1993. Differential expression of multiple fork head related genes during gastrulation and axial pattern formation in the mouse embryo. Development 118: 47-59.

Shim, E.Y., Woodcock, C., and Zaret, K.S. 1998. Nucleosome positioning by the winged helix transcription factor HNF3. Genes \& Dev. 12: 5-10.

Sosa-Pineda, B., Wigle, J.T., and Oliver, G. 2000. Hepatocyte migration during liver development requires Prox1. Nat. Genet. 25: 254-255.

Szebenyi, G. and Fallon, J.F. 1999. Fibroblast growth factors as multifunctional signaling factors. Int. Rev. Cytol. 185: 45106.

Thomas, P.Q., Brown, A., and Beddington, R.S. 1998. Hex: A homeobox gene revealing peri-implantation asymmetry in the mouse embryo and an early transient marker of endothelial cell precursors. Development 125: 85-94.

Waddington, C.H. 1940. Organisers and genes. Cambridge University Press, Cambridge, UK.

Wilson, P.A. and Hemmati-Brivanlou, A. 1995. Induction of epidermis and inhibition of neural fate by BMP-4. Nature 376: 331-333.

Wilson, P.A., Lagna, G., Suzuki, A., and Hemmati-Brivanlou, A. 1997. Concentration-dependent patterning of the Xenopus ectoderm by BMP4 and its signal transducer Smad1. Development 124: 3177-3184. 


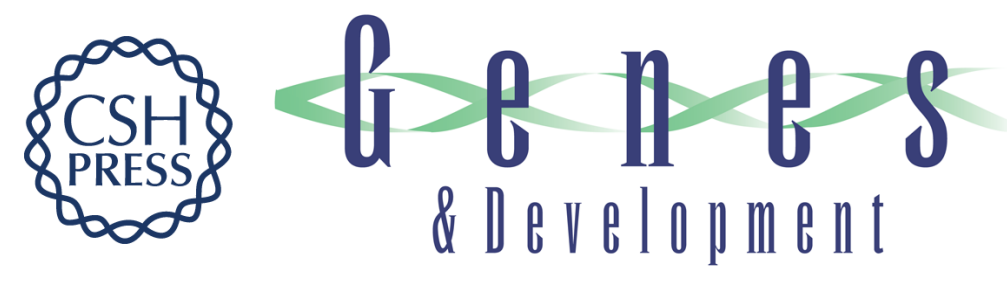

\section{BMPs on the road to hepatogenesis}

Stephen A. Duncan and Alistair J. Watt

Genes Dev. 2001, 15:

Access the most recent version at doi:10.1101/gad.920601

References This article cites 39 articles, 22 of which can be accessed free at: http://genesdev.cshlp.org/content/15/15/1879.full.html\#ref-list-1

License

Email Alerting Receive free email alerts when new articles cite this article - sign up in the box at the top Service right corner of the article or click here.

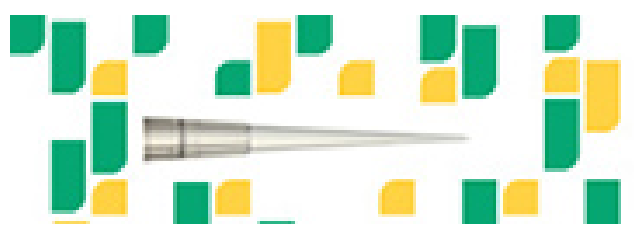

Focused on your science. 